Slide1. Good afternoon, my name is Bilyana Stoilova from Paresh Vyas's lab in Oxford. I would like to thank the organizers for giving me the opportunity to present our work characterizing human hemopoietic lympho-myeloid progenitors using single cell assays.

\title{
Slide2.
}

Human hematopoiesis produces 10 billion blood cells daily. However, there is active debate about the contribution of stem cells and progenitors to steady state hematopoiesis. Research efforts are focused on understanding the molecular mechanisms of normal cell fate decisions but also how genetic and epigenetic changes may lead to differentiation arrest at a progenitor like stage in Acute Myeloid leukaemia, the most common aggressive human leukaemia. In this talk I will focus on human lympho-myeloid progenitors. In the myeloid compartment they make granulocytes and monocytes by not erythroid and Mks cells. Two human lympho-myeloid progenitors have been identified in the early progenitor compartment: the lymphoid-primed multi-potent progenitor (LMPP) identified by our lab, which has granulocytic, monocytic and lymphoid potential and the multi-lymphoid progenitor (MLP) described in John Dick's lab. The MLP unlike the LMPP expresses CD10 and does not have granulocytic potential. Within the late progenitor compartment the granulocyte-macrophage progenitor (GMP) has mainly myeloid but also residual lymphoid potential.

Slide3. What is not clear is whether the human lympho-myeloid progenitors are pure or heterogeneous, what distinguishes them and what are the functional and transcriptional relationships between them.

To answer these questions we performed both population and single cell analyses. We performed in vivo functional analysis using new humanized ossicle assay. We performed bulk in vitro cultures to define the differentiation kinetics and 3 different single cell in vitro assays to assess the functional potential of 4357 single cells. Furthermore, we performed gene expression analysis using population RNA-seq and single cell RNAseq and qRT-PCR. In the interest of time I will show you only the key findings.

Slide4. First, we improved prior FACS strategies to purify eight human HSPC populations in cord blood and bone marrow. Importantly, despite some discrepancies in 
the field, the stem and early progenitor compartment contains a mixture of prospectively separable CD10- LMPP and CD10+ MLP progenitors. LMPPs and MLPs are very rare, each constitute $\sim 1 / 10^{4}-\sim 1 / 10^{5}$ MNCs in CB and BM. On the other hand, GMPs are 10-100-fold more abundant. As the frequency of adult BM LMPP and MLP is extremely low, all assays presented hereafter are performed with fresh CB cells.

Slide5. First we asked what is the differentiation potential of these progenitor populations in vivo. Since human progenitors engraft very poorly, we used a recently described humanized ossicle model, which allowed 10-100 fold better engraftment. LMPP and GMP gave more than 10 times higher engraftment than MLP. Engrafted LMPPs produced more myeloid cells, MLPs - more lymphoid cells, whereas GMP mainly myeloid cells. Of the myeloid cells, LMPP and GMP produced CD15+ granulocytes and CD14+ monocytes. On the other hand the myeloid cells produced by MLP did not express myeloid maturation markers. Therefore, the in vivo assay suggests that LMPP, MLP and GMP progenitor populations have different degrees of lymphoid and myeloid potential.

Slide6. Since we can not transplant single cell human progenitors in vivo, we used 3 different single cell in vitro assays to further characterize them at the clonal level. We first analyzed 3468 single cells in a new efficient liquid culture assay supporting B, NK, Mo and $G$ cell differentiation. In this condition LMPP and GMP had 6-7 times higher cloning efficiency compared to MLP. Focusing on wells that gave single cell cultures the majority of the progenitors gave uni-lineage output. However, rarer bi-lineage and even less frequent multi-lineage outputs were also detected. Note the scales are very different. Summarizing the lineage outputs, MLP was mainly a lymphoid progenitor, while LMPP was lymphoid-biased and GMP myeloid-biased lympho-myeloid progenitor. Importantly, only 7-8\% of all plated LMPPs and GMPs but not MLP had combined lympho-myeloid potential. Similar results were obtained using a published condition from John Dick's lab. 
Slide7. Since the previous assays did not allow looking at $T$ cell potential, we studied 631 single cells in T-lympho-myeloid assay. Again a large diversity of progenitors was detected. T-cell output on its own or combined with other lymphoid outputs was detected at $4-8 \%$ of the positive wells from LMPP and MLP populations and rarely from the GMP. Interestingly, in these conditions lympho-myeloid output was only seen from the LMPP. Overall, in three in vitro single cell assays 24 different progenitor types were detected, all of which were seen in the LMPP and subsets were detected in MLP and GMP.

Slide8. In order to separate functionally distinct progenitors within the heterogeneous LMPP, MLP and GMP populations, which correlate with the single cell functional data, we performed single cell RNAseq of 320 single cells. Hierarchical clustering assigned GMPs, LMPPs and MLPs to three clusters. Based on their gene expression and cell composition, cluster 1 is a lymphoid cluster; cluster 3 is a myeloid cluster and cluster 2 a lympho-myeloid progenitor cluster with high expression of KIT and intermediate expression of lymphoid and myeloid genes.

Principal component analysis showed that LMPP, MLP and GMP form a transcriptional continuum rather than discrete populations.

Slide9. What I didn't mention earlier is that for all the single cell functional experiments we had FACS indexing data, which allowed us to correlate the imunophenotype with the functional output and design new sorting strategies to purify for function. We observed that GMP cells with myeloid output had higher expression of CD38. Therefore, we used a revised sorting strategy based on CD38. Compared to conventional GMPs, GMP CD38hi had significantly lower lymphoid and combined lympho-myeloid potential.

We used a similar strategy for LMPP but this time using CD10 and CD45RA markers, which suggested that LMPPs with high expression of the both markers, called here LMPPly would be depleted of myeloid potential. As expected, in the single cell assay LMPPly cells lacked myeloid potential compared, unlike LMPPmix and conventional LMPP. Therefore the revision of the sorting strategies allowed for purification of more homogeneous populations within the human lympho-myeloid progenitor populations. 
Slide10. To conclude:

- at a single cell level LMPP, MLP and GMP compartments are functionally heterogeneous;

- Single cell transcriptional analysis shows the three populations form a transcriptional continuum.

- FACS indexing data allow refinement of human lympho-myeloid progenitor purification. Our single cell results suggest an alternative hematopoiesis model different from the current one and beyond the borders of conventional immunophenotype. Multiple, rare, functionally distinct lympho-myeloid progenitors or LMPs lie downstream from stem cells and multi-potent progenitors. Lymphoid and myeloid only progenitors are shown underneath. Bi-lineage progenitors are more frequent and uni-lineage progenitors are most common. The hierarchical relationships between these progenitors remain to be determined.

Slide11. Main people involved in the project are Dimitris Karamitros and Zahra Aboukhalil from the Vyas lab, Fiona Hamey from Gottgen lab and Andreas Reinsch from Majeti lab. Thank you for your attention and I am happy to take questions.

Potential questions:

What are the 85 genes differentially expressed between LMPP and MLP

- These include some regulators of $\mathrm{NOTCH}$ signaling, Zn-finger proteins, GPCRs, signaling molecules. However, I must mention that when we used some of these genes for qRT-PCR the data were quite noisy with quite some variation between the cells, which can be expected. But if we plotted the average of all single cells, we observed the same pattern like population RNAseq.

What are the myeloid cells produced by MLP

- Thank you for this question. We were also quite curious what they were. We did some differentiation kinetics to see whether at different time point we might catch mature myeloid cells from MLP, since data here are from week 2 post transplant. When we looked at week 1, we saw only myeloid cells coming from LMPP, MLP, GMP and no lymphoid cells yet. These myeloid cells were mainly neutrophils and a few monocytes. 
The reason we decided to report week 2 is because of both lymphoid and myeloid graft, which can be detected only at week 2 .

How many cells were transplanted

- Fresh CB - up to 2 days old; irradiation; 5.000-30.000 cells transplanted;

What are the CD14+CD15+DP cells

- We think these are less mature cells of the neutrophil lineage, since we did some experiments, where we sorted the cells and analysed them in vitro after 3 days. For this period of time it seems that DPs give CD15+ neutrophils.

Why do we observe differences from J. Dick's publication

- This is an excellent question. To be fare, I don't have a clear explanation of this. I guess it might be also somehow related to the fact that JohnDick's lab does not see any CD34+CD38-CD45RA+CD10- cells but only CD10+, while we see a mixture of both and we separate them to study their functions.

Why LMPP is mainly a Ly progenitor in vitro and mainly a My progenitor in vivo

The humanized ossicles may be particularly efficient at promoting human myelopoiesis unlike naive NSG mouse, which better support human lymphopoiesis.

What are the genes expressed by the Ly, My and LM clusters

- Ly-genes: CD3E, TCF7, NOTCH3, CD79A, EBF1

- My-genes: CSF1R, CSF3R, PRTN3, CEBPA, GFI1, MPO

What are the genes separating LMPP and MLP from the rest of HSPCs

- CD3E, IGHD, IGHM, IGHV..., MME, MZB1, NOTCH3, TRBV28

- LMPP, MLP, GMP - IGJ, IRF8

How could you justify the use of $C B$ instead of $B M$

- $\mathrm{CB}-30 \%$ multilin, $\mathrm{BM}-20 \%$ multinil

- in the CD34+CD38- - CB - 45\% multilin, BM - 35\% multilin

- in the CD34+CD38+ - CB - 20\% multilin, BM - 10\% multilin

In our system - 7-9\% LM prog from LMPP and GMP, if we consider the Ly and My only bi-lin prog - 20-25\%

Although there is about $10 \%$ lower multilin progenitors in $B M$ compared to $C B$, when looking at the functional outputs of these progenitors, $C B$ and BM are actually very similar and quite different from FL. I also want to remind you that while John D. is looking at My-Er-Mk progenitors, we are looking at Ly-My progenitors. 
if there is transcriptional continuum, why do you want to define hierarchical relationships - I don't think that the fact that there is a transcriptional continuum stops it from being hierarchical, I would say just the opposite - cells transition from one to another. Also, many of the hierarchical relationships in hematopoiesis are assumed based on potential. We are very keen to show these relationships, but this won't be easy. I can imagine an experiment combining life single cell tracking for a few days and single cell functional assay to define the potential.

What are the genes from the LM cluster in the SC RNAseq

-many enzymes and metabolic genes. As we know metabolism has been previously shown related to stemness, potentially also to early progenitor function, so this might not be surprising.

CD10=MME - CD10: A cell surface enzyme with neutral metalloendopeptidase activity. CD10 is also known as CALLA (common acute lymphocytic leukemia antigen). CD10/CALLA is normally present on the surface of early lymphoid cells.

\section{EHA questions:}

What conditions did you use for the in vitro culture?

3 different conditions for the single cell in vitro cultures

How exactly is the in vivo model you used generated?

In vitro expanded human BM-MSCs were mixed with matrigel matrix. The whole matrixcell mixtures were injected subcutaneously to generate humanized ossicle niches. 8-10 weeks post BM-MSC application transplants were evaluated for bone and marrow formation.

How do you characterize the MSC cells you are using for the model?

BM-MNCs were plated in a-MEM containing $10 \%$ pooled human platelet lysate (pHPL). Nonadherent cells were removed by rinsing the plastic with PBS, and adherent cells were further expanded. Emerging colonies (CFU-F) were passaged for further cell propagation with half-weekly medium changes until the cells reached confluence. At the end of passage $1(\mathrm{p} 1)$, BM-MSCs were profiled for the expression of consensus MSC surface markers: positive - CD90, CD73, CD105; negative - CD45, CD14, CD34, CD19, HLA-DR, CD29. In vitro differentiation: osteoblasts, adipocytes, chondroblasts. 
Do you think the markers we are using to purify these populations are the best ones? Definitely not. As I showed you sorting on the conventional markers results in significant inter-population heterogeneity. Further purification based on the levels of expression of these markers might give better separation. But it is entirely possible that there are other markers which will allow even further purification.

There is a series of papers i.e. Velten et al. etc that describe slightly different models of haemopoiesis how do they fit with your model?

Velten model is quite similar - continuum of differentiation: 'continuum of low-primed undifferentiated haematopoietic stem and progenitor cells' (CLOUD-HSPCs), from which unilineage-restricted cells emerge directly.

John Dick's model - looking at Ery/Mk/My differentiation - mainly uni-lineage progenitors; Ido Amit's model - mouse CMP, GMP, MEP - only uni-potent based on transcriptome - 18 uni-lineage progenitors.

And in Paresh's section they asked why the difference in functional output of LMPP in vivo and in vitro

Single cell vs population assay. The LMPP produces mainly myeloid cells in vivo, suggesting that the humanized ossicles may be particularly efficient at promoting human myelopoiesis, unlike naive NSG mice, which better support human lymphopoiesis. And why did you use only Cord Blood and what about bone marrow?

All our functional and transcriptional analyses were performed on $\mathrm{CB}$. Though the LMPP, MLP and GMP have not been studied in detail through development, quantitative differences in multi- versus uni-lineage output have been documented in My/Ery/Mk in vitro assays between fetal liver and bone marrow in the broad CD34+CD38 \pm populations 